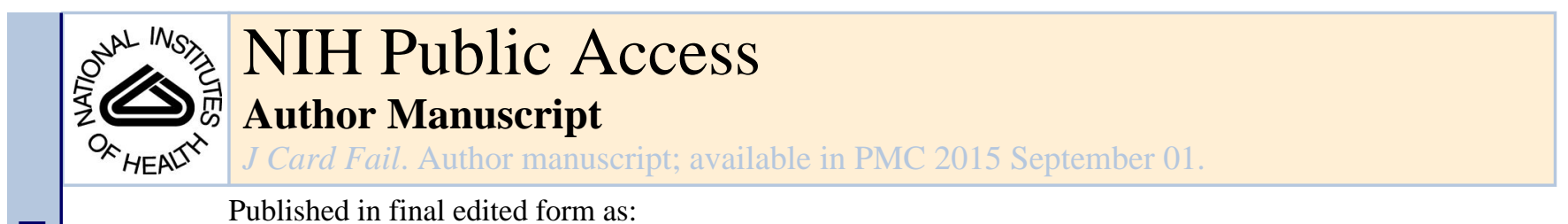

Published in final edited form as:

J Card Fail. 2014 September ; 20(9): 641-649. doi:10.1016/j.cardfail.2014.06.358.

\title{
Joint Commission Requirements for Discharge Instructions in Patients with Heart Failure: Is Understanding Important for Preventing Readmissions?
}

\author{
Ricky Regalbuto, MD¹, Mathew S. Maurer, MD¹, David Chapel, BS ${ }^{1}$, Jenniliz Mendez, BA ${ }^{1}$, \\ and Jonathan A. Shaffer, PhD MS ${ }^{1}$ \\ ${ }^{1}$ Columbia University Medical Center, NewYork Presbyterian Hospital, New York, NY, 10032 USA
}

\begin{abstract}
Background-Many approaches have been considered to reduce heart failure (HF) readmissions. The Joint Commission (JCO) requires hospitals to provide patients admitted for HF with discharge instructions that address six topics related to HF management: diet, exercise, weight monitoring, worsening symptoms, medications, and follow up appointments. These guidelines were developed based on expert opinion, but no one has tested whether patients' understanding of these instructions affects 30-day readmission rates.
\end{abstract}

Methods and Results-We conducted a prospective cohort study of patients admitted for decompensated HF. Patients completed an understanding survey immediately after their nurse read their discharge papers. The survey contained one question for each of the six JCO topics. Of the 145 patients in the study, only 14 (10\%) understood all 6 discharge instructions. Patients with complete comprehension of their discharge instructions were significantly less likely to be readmitted within 30 days than those with non-perfect understanding $(\mathrm{p}=0.044)$, but this association was no longer significant after controlling for level of education and use of English as a primary language.

Conclusions-HF patients' comprehension of discharge instructions is inadequate. Patients with limited education and those that do not speak English as a primary language are more likely to have poorer discharge understanding and higher rates of 30-day readmissions.

\section{Keywords}

Discharge; Readmission; HF-1

(C) 2014 Elsevier Inc. All rights reserved.

Address for Correspondence: Ricky Regalbuto Department of Medicine Columbia University Medical Center NewYork Presbyterian Hospital Milstein Hospital Building 6C-12 177 Fort Washington Ave New York, NY, 10032 212-342-1674 jrr2155@ columbia.edu. Publisher's Disclaimer: This is a PDF file of an unedited manuscript that has been accepted for publication. As a service to our customers we are providing this early version of the manuscript. The manuscript will undergo copyediting, typesetting, and review of the resulting proof before it is published in its final citable form. Please note that during the production process errors may be discovered which could affect the content, and all legal disclaimers that apply to the journal pertain.

Disclosures

None 


\section{Introduction}

Given the multitude of public health and policy implications associated with hospital readmissions $(1,2)$, considerable attention has been paid to developing strategies that keep patients from returning to the hospital. Heart failure (HF) patients, a chronically ill population whose prevalence is increasing (3), are frequently readmitted to the hospital. The national 30-day readmission rate for Medicare patients admitted for HF in 2010 was 24.6\% (4), essentially unchanged from the previous two years, and among all Medicare patients those with HF are more likely than any other patient group to be readmitted (2). There has been considerable effort to lower this readmission rate as part of broader health care reform, and Medicare has begun to inflict financial penalties on hospitals that have excessive readmission rates (1).

Because HF readmissions are common, multiple approaches have been considered to reduce them. Some hospitals have used education classes (5) while others have piloted programs that involve having nurses follow patients in their homes (6). One national initiative that was begun in 2002 is the Joint Commission's (JCO) standard for discharge instructions $(7,8)$. This standard, known as HF-1, requires that all discharge instructions address 6 specific areas related to HF management: activity level, diet, discharge medications, follow-up appointments, weight monitoring, and procedures to follow if symptoms worsen (7). Although this standard was developed based solely on expert opinion, previous research has shown that among $782 \mathrm{HF}$ patients, those who received instructions that adhered to these JCO guidelines were less likely to be readmitted within one year of discharge than those who did not (9). This study, though suggestive of the importance of HF-1, was limited by its retrospective nature and focus on 1-year readmissions as a primary outcome, which is a distal endpoint for assessing the efficacy of discharge instructions. Additionally, there was no attempt made to determine if patients understood the instructions they were provided. Although ensuring that all patients receive quality instructions is an appropriate initial step, doing so offers little help if they do not understand these instructions. Indeed, multiple studies $(5,10,11)$ have shown the importance of education, symptom understanding, and self-care in promoting medication adherence, reducing care costs, and preventing readmissions among HF patients.

Our primary aim for the current study was to identify demographic and clinical characteristics that predict HF patients' understanding of their discharge instructions. Given the recent focus on reducing 30-day readmissions after HF hospitalization, we further sought to study the association of patients' understanding of JCO-approved discharge instructions on 30-day hospital readmissions. We hypothesized that greater understanding of discharge instructions would be associated with lower 30-day rates of readmission.

\section{Methods}

\section{Study Population}

Eligible participants included all patients discharged from NewYork Presbyterian Hospital/ Columbia University Medical Center from August 1, 2012 to November 19, 2012 with a primary admitting diagnosis of decompensated HF. The HF exacerbation diagnosis, along 
with the absence of any excluding criteria, was confirmed by study personnel by speaking to the physician team responsible for each patient's care and by consulting the medical record. Participants were eligible if the primary reason for admission was HF and were not eligible if they had a history of dementia, were going to be discharged to a location other than home, did not speak either English or Spanish, had ever received a heart transplant or left ventricular assist device (LVAD), or if their HF was due to a congenital heart defect. As shown in Figure 1, 181 of the 237 eligible patients were enrolled. The study was approved by the institutional review board of Columbia University Medical Center, and all patients provided informed consent to participate.

\section{Demographic and Clinical Characteristics}

Demographic and clinical data were obtained from several sources. Patients completed a demographic survey that was administered immediately after enrollment in the study, approximately one to two days prior to discharge. This survey included questions about racial and ethnic background, monthly income, level of educational attainment, and marital status. It also included the two-item Patient Health Questionnaire (PHQ-2), a validated assessment of depression (12). Other clinical variables including echocardiographic parameters (e.g. ejection fraction) and laboratory data (e.g. serum $\mathrm{Na}$, creatinine, and hemoglobin) were obtained during hospitalization from the electronic medical record.

\section{Assessment of Understanding of JCO Discharge Instructions}

Patients were assessed for their understanding of their discharge instructions by means of an understanding survey. The survey was developed to provide an assessment of how well patients understood the six parts of discharge instructions that are included in HF-1. The study authors worked with an adult health literacy expert to develop the survey instrument, with a particular focus on ensuring the survey would be concise while providing an accurate representation of a patient's discharge understanding. Multiple-choice questions were used to ask about discharge diet, exercise regimen, weight monitoring and what a patient would do if symptoms returned. For the questions about follow-up appointments and discharge medications, patients were asked to list their appointments and medication regimens. The study was pilot tested on 10 patients, and the results were used to determine how the instrument would be scored during the study. This understanding survey was administered immediately after each patient's nurse read the standardized discharge instructions, which included a medication list and a list of all follow-up appointments. For patients that did not speak English, discharge nurses used a phone interpreter service as per hospital protocol. For all surveys, patients had the option to read and complete the surveys on their own or to have the questions read aloud to them and have their answers marked. Survey materials were administered in both English and Spanish, and patients had the option of using either language. All study materials were translated by a native Spanish speaker with experience in translating materials for research studies.

\section{Statistical Analyses}

Responses to the understanding survey were scored by summing the responses to each item ( 0 or 1 ), yielding a total score ranging from 0 to 6 . Based on the results of pilot testing, answers were judged to be correct if patients responded with an answer that corresponded 
with national guidelines (13) or that matched an alternate instruction that was printed on their discharge paperwork. The understanding survey used in the study, along with a more detailed explanation of its scoring, is presented in the Appendix.

To ascertain 30-day readmissions we first used the electronic medical record to see if a patient was readmitted to the hospital and/or whether they had attended their scheduled follow-up appointment. All patients were also contacted by telephone 30 days after discharge to further determine recurrent hospitalizations. All phone calls followed a standard script. If patients did not answer their phone, multiple attempts were made on the subsequent days until stopping at day 35 . Additionally, as part of routine hospital policy, all patients were called within 3 days of discharge by nursing staff. We recorded whether or not a patient answered this 3-day phone call.

Multivariable linear regression analyses were conducted to examine which clinical and demographic factors predicted understanding. These clinical and demographic factors were chosen based on univariate analyses of factors that might predict understanding. Patients' understanding scores were treated as a continuous variable. Kaplan-Meier (KM) survival analysis was conducted to determine whether time-to-readmission varied as a function of understanding, and the log-rank test was used to test statistical significance. Given the results of these primary analyses, we conducted two post hoc KM survival analyses to test whether education level and primary language predicted 30-day readmissions, a post hoc Cox proportional hazards survival analysis predicting 30-day readmission from age, education level, primary language, and understanding, and a series of chi-square analyses testing the association of receipt of a 3-day post-discharge phone call with readmission status, understanding, education, and language. For the KM analyses, we treated understanding as a binary variable with categories of complete understanding (score of 6) and incomplete understanding (score $\leq 5$ ). All data were analyzed using SPSS software (IBM, Armonk, NY).

\section{Results}

Figure 1 shows the flow of study recruitment. In total, 145 patients successfully completed the study. The baseline demographics of these 145 patients are shown in Table 1. Our cohort consisted of an older, multicultural population, with multiple medical comorbidities. The characteristics of this group are consistent with other trials involving patients hospitalized for acute HF exacerbations (14-16).

Readmission and covariate data were collected for all 145 patients that completed the study. All but one patient received discharge instructions that addressed the 6 areas required by JCO. Patients' understanding of their discharge instructions varied. On average, the 145 patients answered $4.1( \pm 1.2)$ questions correctly on the understanding survey. Table 2 shows the average understanding score and the breakdown of individual scores. As seen in Figure 2, patients performed best on the question about what to do if their symptoms worsened and poorest on the question that asked about their medications, with less than $20 \%$ of patients able to name all of their medications correctly. Among all participants, the mean understanding score for English speakers was $4.33(\mathrm{SD}=1.11)$ and for non-English speakers 
was $3.44(\mathrm{SD}=1.04, \mathrm{p}<0.001)$. Further, the mean understanding for patients with a college education or greater was $4.58(\mathrm{SD}=0.92)$ and for patients with a high school education or less was $3.79(\mathrm{SD}=1.18), \mathrm{p}<0.001)$.

To identify clinical and demographic factors that predict patients' understanding of their discharge instructions, a multivariable linear regression analysis was performed. As shown in Table 3, both language spoken and educational attainment were significant predictors of discharge comprehension. In the multivariable analysis, the understanding score of Englishspeaking patients was 0.9 points greater than that of patients who did not speak English $(\mathrm{p}=$ 0.009 ). Similarly, having completed a college education was associated with a 0.6 point increased understanding score $(\mathrm{p}=0.012)$. Understanding score was not significantly predicted by ethnicity or race, sex or age. The service to which patients were admitted for their hospitalization also did not predict the understanding score. Notably, only $22 \%$ of the variance in understanding scores was explained by the variables included in the multivariate analysis.

Consistent with national figures (4), 34 of 145 patients (23.4\%) were readmitted to a hospital within 30 days. To determine whether level of understanding (complete versus incomplete) predicted time-to-readmission, we performed a KM survival analysis for all patients $(\mathrm{n}=130)$ that completed follow-up (Figure 3$)$. No patients with a perfect understanding score were readmitted within 30 days, and thus we observed a significant difference in time-to-readmission among patients with incomplete understanding compared to those with complete understanding $(\mathrm{p}=0.044)$. Because education and language predicted greater discharge understanding, we also performed KM analyses to test whether time to readmission varied as a function of education level and primary language (Figures 4 and 5). We observed a significant difference in time-to-readmission between patients who had a college degree compared to those who did not $(\mathrm{p}=0.023)$ and for patients who spoke English as a primary language compared to those who did not $(p=0.039)$. The hazard ratios for 30-day readmission for patients without a college degree and for those who did not speak English as a primary language were $3.1(\mathrm{p}=0.022)$ and $2.2(\mathrm{p}=0.052)$ respectively. After performing a Cox proportional hazards survival analysis controlling for age, education level, and language spoken, the understanding score no longer significantly predicted 30-day readmission $(p=0.97)$. A final post hoc analysis, which assessed the impact of a patient answering the 3-day nursing follow-up phone call, found no association of receipt of a 3-day phone call with readmission status, understanding, education, or language.

\section{Discussion}

We found that HF patients' understanding of their discharge instructions is limited, particularly with respect to their knowledge of follow-up appointments and discharge medications, despite receiving discharge instructions that adhere to JCO guidelines. This study is the first that we are aware of to specifically examine how well discharge instructions for HF that meet JCO guidelines are understood by patients in a naturalistic setting and how comprehension of these discharge instructions impacts 30-day rates of readmission. Our findings build upon previous studies, both qualitative (17) and quantitative 
$(18,19)$, that have shown that patients with HF have a difficult time understanding their disease and medications.

A study by $\mathrm{Ni}$ and colleagues (19) of $113 \mathrm{HF}$ patients found that, depending on the wording of items in a survey, their cohort was able to recognize the importance of a low sodium diet $76 \%$ to $90 \%$ of the time. This finding is comparable to the $81 \%$ of patients in our study who correctly identified the importance of a heart healthy diet. Patients in our group had a better understanding than those in the Ni study of the importance of daily weight monitoring, with over $80 \%$ responding correctly compared to only $60 \%$ in the Ni cohort. Importantly, the cohort in the Ni study was composed of outpatients attending their first visit to a tertiary care HF clinic, without any specific intervention beforehand. In contrast, our cohort contained acutely ill inpatients that had just been read detailed discharge instructions by a nurse. This difference likely explains why our cohort performed better when asked about weight monitoring and just as well when asked about diet, despite the fact that as a group they were less educated than the Ni cohort. Our study also found that less educated patients had a poorer understanding of their discharge instructions, a finding that is similar to work done by Artinian and colleagues (18) in an outpatient setting.

Neither the Ni nor Artinian studies looked at discharge medication understanding, whereas we did so as part of our assessment of JCO's HF-1 guideline. Patients in our study were discharged on an average of ten medications, and very few (18\%) correctly named all of their discharge medications. Other groups that have looked at medication adherence after hospitalization have also found that patients have a difficult time with medication understanding. One study by Maniaci and colleagues (20), for instance, showed that when asked within 18 days of discharge to name new medications started during a hospital visit, only $64 \%$ of patients could do so. Another study by Cline and collaborators (21) found that only $55 \%$ of elderly HF patients who were interviewed 30 days after leaving the hospital could report new medications that had been added to their regimen. The fact that our cohort did much more poorly than patients from these two previous studies likely reflects the fact that we asked patients to name all of their medications, not just the new ones. Even more alarming was the fact that we allowed all patients to review their discharge instructions to help them answer our survey. While this provision seemingly would have allowed patients to just read off the answers, it proved to be very difficult, as patients were confused by the medical language and multiple lists of medicines that were on the discharge papers. As this finding shows, just handing patients a medication list as part of a large packet of instructions, the minimum required by HF-1, will likely do little to improve medication understanding. Collectively these data suggest that ensuring understanding of medication regimens at discharge merits special attention, as poor understanding is a known barrier to medication adherence (22).

While it is certainly important and noteworthy to assess what aspects of discharge instructions patients understand, our data also show that understanding alone is not an independent predictor of readmission when controlling for language and level of education. Furthermore, both primary language and education level are predictors of readmission. This finding is novel for HF patients, and it builds upon research on general medicine patients and population level studies (23-25). Our study shows that patients who do not speak 
English have a 2.2-fold higher likelihood of being readmitted $(p=0.052)$ than those who speak English. Similar to our findings, previous studies $(24,25)$ not specific to HF have found that general medicine patients who speak Spanish have higher rates of 30-day readmission ( $\mathrm{OR}=1.5,95 \% \mathrm{CI}=1.0-2.3)$ and are less likely to understand their discharge follow-up appointments and discharge medications. Although no studies have assessed the efficacy of using trained in-person translators in the discharge setting, the use of such personnel may improve understanding of discharge instructions for non-English speakers.

Our study also found that patients with less than a college education had a 3.1-fold higher likelihood of being readmitted within 30 days $(p=0.022)$ compared to those with a college education or higher, and that these patients were more likely to have poor discharge understanding. Arbaje et al (23) found that among all Medicare patients, those without a high school diploma were more likely to be readmitted within 60 days than those with a high school diploma $(\mathrm{OR}=1.4,95 \% \mathrm{CI}=1.01-2.02)$. This finding suggests that an approach tailored to those with lower health literacy may improve readmission outcomes. One modification specific to the discharge process that may prove helpful fin reducing readmissions is the use of discharge materials written at reading levels appropriate for a broad range of patients. Work done in emergency departments has repeatedly found that discharge instructions are often written above patients' reading levels, using medical language that is difficult to understand (26-29).

While no patients who had complete understanding of their discharge instructions returned to the hospital within 30 days, perfect understanding of discharge instructions did not independently predict readmission once education and language were controlled. This result implies that greater comprehension of the discharge instructions proscribed by HF-1, in and of itself, is not sufficient to significantly reduce readmissions. Previous studies that have examined discharge interventions and found a reduction in readmissions have utilized interventions that went beyond what is required in HF-1. Koelling and colleagues, for instance, found that a one-on-one, hour-long teaching and counseling session about HF delivered by a nurse educator prior to discharge reduced 6-month HF rehospitalization rates by half (10). Similarly, a meta-analysis by Phillips and colleagues found that comprehensive discharge planning (including one-on-one teaching sessions, geriatric discharge protocols, and home care coordination) combined with post-discharge support resulted in a $25 \%$ relative reduction in readmissions over 3 to 12 months after discharge among 18 studies with 3,304 participants (30). Notably, the interventions studied in the meta-analysis did not rely on discharge education alone to reduce readmission rates; rather, they almost all employed telephone or home visits to ensure close follow up. Although it is difficult to parse out which aspects of these interventions were most responsible for the reductions in readmissions, the consistent association of increased HF understanding with lower readmissions observed in the Koelling study and the Phillips meta-analysis suggests that the success of discharge intervention on reducing readmissions may require a comprehensive program that goes beyond merely providing patients with a discharge instruction sheet.

Although previous work by Harrison and colleagues (31) showed that receipt of a single post-discharge phone call may reduce rates of readmission, a post hoc analysis of our data failed to replicate this finding. This discrepancy in our findings compared to those of 
Harrison and colleagues may be attributable to the smaller sample size in our study compared to the Harrison study $(n=30,272)$. Future JCO initiatives to improve the process of HF discharge could include broader implementation of the types of one-on-one teaching and post-discharge support programs that have been shown to reduce readmissions.

Notably, only $22 \%$ of the variance in understanding scores was explained by the factors we measured in our study, suggesting that there are factors associated with discharge understanding that we did not capture. We hypothesize that these unmeasured variables may include which specific physician and nurse were taking care of each patient. Certain doctors may devote additional time to ensure that patients understand their post-discharge plan, and certain nurses may be more experienced at delivering discharge instructions. While this issue was not actively pursued in this study, it represents a possible avenue for future research.

In contrast to previous work, our study focused exclusively on 30-day readmissions as opposed to readmission over longer follow-up periods. Although there is considerable debate over the value of 30-day readmissions as a metric by which to judge hospitals (32), especially when over $50 \%$ of readmissions are not for HF (2), it seems to be a useful time frame to evaluate the effectiveness of discharge interventions, as these 30 days may be critical ones during which patients are especially vulnerable to being readmitted (33). Despite the widespread use of HF-1, data about its efficacy has been limited. The previous work assessing the efficacy of HF-1 used a 12-month time span to track readmissions (8), and our study is the first to our knowledge to show that complete understanding of the information contained in HF-1 is not an independent predictor of readmission in the immediate 30-day period following discharge.

The strengths of our study include its prospective design and the comprehensive follow-up data we obtained by using both the medical record and making follow-up phone calls after 30 days. This procedure ensured that we were able to capture patients who were readmitted to other hospitals, whom we would have missed if we had relied solely on the medical record from our institution. One other novel aspect of our study was its inclusion of patients who spoke primarily Spanish, which proved important, as their understanding of their discharge directions and rates of readmission were significantly poorer than English speakers. Limitations of the study include the relatively small sample size, and the possibility that our survey instrument may not have been an effective tool to capture patients understanding. Although the validity of our instrument to assess understanding has not been previously evaluated for its psychometric properties, we developed the tool with input from health literacy experts to help ensure it would be an accurate representation of patients' knowledge.

\section{Conclusion}

In this diverse, multi-ethnic urban cohort of HF patients, those with a complete understanding of their JCO-compliant discharge instructions were significantly less likely to be readmitted to the hospital within 30 days than those with a less than perfect understanding, but this association was no longer significant after controlling for education 
level and use of English as a primary language. Having a college education and being able to speak English independently predicted greater understanding of discharge instructions. There remains a great deal of potential improvement needed to increase HF patients' understanding of their discharge instructions, particularly concerning medications, which may be addressed by modifications to JCO guidelines.

\section{Acknowledgments}

We would like to thank the attending physicians, residents, nursing staff and care coordinators of 5GS, 5GN and 7GS at the Columbia University Medical Center for their help in enrolling patients. We would also like to thank Emelin Martinez for her help in devising our survey instrument, Oscar Peña for his work translating our consent forms, Dr. Carol Ludwig for funding the Sara and Arnold P. Friedman Award that allowed us to present an abstract of this work at the 2013 ACC meeting.

\section{Appendix 1: Understanding Survey and Detailed Scoring Methodology}

Circle the one sentence that you think is true about exercise and your heart.
a. Exercise may be harmful for my heart and I should wait before starting
b. Exercising 30 minutes five times per week is beneficial or good for my heart
c. I should exercise as much as I can tolerate
d. Exercise will have no effect on my heart
e. I do not know how exercise will affect my heart

Circle the one sentence that you think is the most important advice to follow about your diet.
a. I should eat plenty of fruits and vegetables
b. I should limit how much fat I eat
c. I should limit how much salt I eat and water I drink
d. I should drink more water
e. I do not know the most important advice to follow about my diet

When should you check your weight? Circle one answer.
a. every day at home
b. every week at home
c. every month at home
d. whenever I go to the doctor
e. never
f. I do not know how often my weight should be checked

What should you do if your symptoms return or get worse? Circle one answer.
a. wait a few days and see if I feel better
b. stop taking my medications 
c. call my doctor or 911

d. I do not know what to do if my symptoms return or get worse

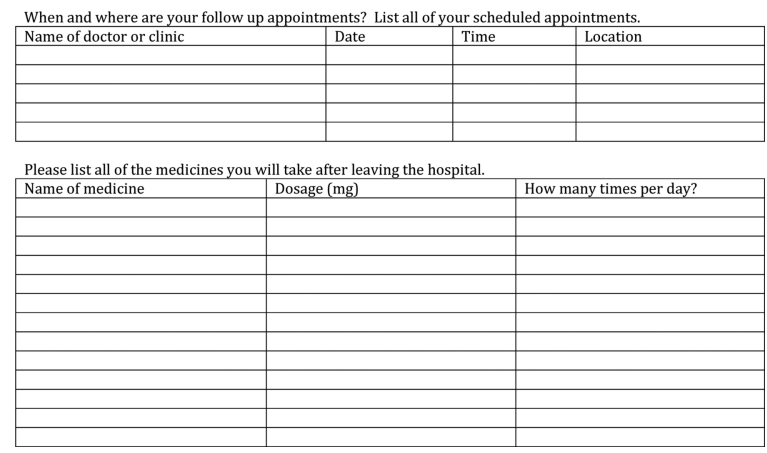

For the exercise question patients received a point if they correctly identified that exercising for 30 minutes five times per week is beneficial for their heart or if they identified that they should wait before exercising (for patients who had recently had an MI or a major procedure performed, such as catheterization with stenting or $\mathrm{CABG}$ ). Patients also received a point for the exercise question if they matched alternate instructions given on their discharge papers, such as that they should exercise as much as tolerated. For the diet question, patients received a point if they identified that they should follow a low salt diet or they were able to match alternate dietary advice (e.g., eating fruits and vegetables) provided on the discharge summary. For the question about weight, patients received a point if they chose that they should check their weight daily. For the question regarding responses to worsening symptoms, patients received a point for responding that they would call their doctor or 911 in the case of worsening symptoms. For the question about follow-up appointments, patients scored a point if they were able to correctly list all of the names of the doctors or clinics at which they had follow up visits scheduled. For the medications question, patients scored a point if they were able to correctly list all of the medicines they would be taking after they left the hospital. For all of these questions, patients' actual discharge summaries and discharge medication lists were accessed from the electronic medical record and used to score each item. All decisions about how the instrument would be scored were made before the investigators began the study.

\section{References}

1. Foster D, Harkness G. Healthcare reform: pending changes to reimbursement for 30-day readmissions. Thomson Reuters. 2010

2. Jencks SF, Williams MV, Coleman EA. Rehospitalizations among patients in the Medicare fee-forservice program. New England Journal of Medicine. 2009; 360(14):1418-28. [PubMed: 19339721]

3. Bui AL, Horwich TB, Fonarow GC. Epidemiology and risk profile of heart failure. Nature reviews Cardiology. Jan; 2011 8(1):30-41.

4. Suter, Lisa G.; Vellanky, Smitha; Li, Shu-Xia; Strait, Kelly; Eddy, Elizabeth; Okai, Meechen, et al. Medicare Hospital Quality Chartbook 2012: Performance Report on Outcome Measures. Yale New Haven Health Services Corporation Center for Outcomes Research and Evaluation. 2012

5. Krumholz HM, Amatruda J, Smith GL, Mattera JA, Roumanis SA, Radford MJ, et al. Randomized trial of an education and support intervention to preventreadmission of patients with heart failure. Journal of the American College of Cardiology. 2002; 39(1):83-9. [PubMed: 11755291] 
6. Naylor MD, Brooten DA, Campbell RL, Maislin G, McCauley KM, Schwartz JS. Transitional care of older adults hospitalized with heart failure: a randomized, controlled trial. Journal of the American Geriatrics Society. 2004; 52(5):675-84. [PubMed: 15086645]

7. HF-1. [June 5, 2013] Specifications Manual for National Hospital Inpatient Quality Measures [Internet]. The Joint Commission. 2013. [HF-1]. Available from: http://www.jointcommission.org/ specifications_manual_for_national_hospital_inpatient_quality_measures.aspx

8. Core Measure Sets: The Joint Commission. [2013 June 5] 2013. [Website]. Available from: http:// www.jointcommission.org/core_measure_sets.aspx

9. VanSuch M, Naessens JM, Stroebel RJ, Huddleston JM, Williams AR. Effect of discharge instructions on readmission of hospitalised patients with heart failure: do all of the Joint Commission on Accreditation of Healthcare Organizations heart failure core measures reflect better care? Quality \& Safety in Health Care. Dec. 2006; 15(6):414-7. [PubMed: 17142589]

10. Koelling TM, Johnson ML, Cody RJ, Aaronson KD. Discharge education improves clinical outcomes in patients with chronic heart failure. Circulation. Jan 18. 2005; 111(2):179-85. [PubMed: 15642765]

11. Wu JR, Moser DK, Lennie TA, Peden AR, Chen YC, Heo S. Factors influencing medication adherence in patients with heart failure. Heart \& Lung : The Journal of Critical Care. Jan-Feb. 2008; 37(1):8-16. e1. [PubMed: 18206522]

12. Kroenke K, Spitzer RL, Williams JBW. The Patient Health Questionnaire-2: Validity of a TwoItem Depression Screener. Medical Care. 2003; 41(11):1284-92. [PubMed: 14583691]

13. Lindenfeld J, Albert NM, Boehmer JP, Collins SP, Ezekowitz JA, Givertz MM, et al. Executive Summary: HFSA 2010 Comprehensive Heart Failure Practice Guideline. Journal of Cardiac Failure. 2010; 16(6):475-539.

14. Felker GM, Lee KL, Bull DA, Redfield MM, Stevenson LW, Goldsmith SR, et al. Diuretic strategies in patients with acute decompensated heart failure. New England Journal of Medicine. 2011; 364(9):797-805. [PubMed: 21366472]

15. O'Connor C, Starling R, Hernandez A, Armstrong P, Dickstein K, Hasselblad V, et al. Effect of nesiritide in patients with acute decompensated heart failure. New England Journal of Medicine. 2011; 365(1):32-43. [PubMed: 21732835]

16. Teerlink JR, Metra M, Felker GM, Ponikowski P, Voors AA, Weatherley BD, et al. Relaxin for the treatment of patients with acute heart failure (Pre-RELAX-AHF): a multicentre, randomised, placebo-controlled, parallel-group, dose-finding phase IIb study. The Lancet. 2009; 373(9673): 1429-39.

17. Rogers A, Addington-Hall JM, McCoy AS, Edmonds PM, Abery AJ, Coats AJ, et al. A qualitative study of chronic heart failure patients' understanding of their symptoms and drug therapy. European Journal of Heart Failure. 2002; 4(3):283-7. [PubMed: 12034153]

18. Artinian NT, Magnan M, Christian W, Lange MP. What do patients know about their heart failure? Applied nursing research. Nov. 2002; 15(4):200-8. [PubMed: 12444578]

19. Ni H, Nauman D, Burgess D, Wise K, Crispell K, Hershberger RE. Factors influencing knowledge of and adherence to self-care among patients with heart failure. Archives of Internal Medicine. 1999; 159(14):1613. [PubMed: 10421285]

20. Maniaci MJ, Heckman MG, Dawson NL. Functional Health Literacy and Understanding of Medications at Discharge. Mayo Clinic Proceedings. 2008; 83(5):554-8. [PubMed: 18452685]

21. Cline CMJ, Björck-Linné AK, Israelsson BYA, Willenheimer RB, Erhardt LR. Non-compliance and knowledge of prescribed medication in elderly patients with heart failure. European Journal of Heart Failure. 1999; 1(2):145-9. [PubMed: 10937924]

22. Kripalani S, Henderson LE, Jacobson TA, Vaccarino V. Medication Use Among Inner-City Patients After Hospital Discharge: Patient-Reported Barriers and Solutions. Mayo Clinic Proceedings. 2008; 83(5):529-35. [PubMed: 18452681]

23. Arbaje AI, Wolff JL, Yu Q, Powe NR, Anderson GF, Boult C. Postdischarge Environmental and Socioeconomic Factors and the Likelihood of Early Hospital Readmission Among CommunityDwelling Medicare Beneficiaries. The Gerontologist. Aug 1; 2008 48(4):495-504. 2008. [PubMed: 18728299] 
24. Karliner LS, Auerbach A, Nápoles A, Schillinger D, Nickleach D, Pérez-Stable EJ. Language barriers and understanding of hospital discharge instructions. Medical Care. 2012; 50(4):283. [PubMed: 22411441]

25. Karliner LS, Kim SE, Meltzer DO, Auerbach AD. Influence of language barriers on outcomes of hospital care for general medicine inpatients. Journal of Hospital Medicine. 2010; 5(5):276-82. [PubMed: 20533573]

26. Chacon D, Kissoon N, Rich S. Education attainment level of caregivers versus readability level of written instructions in a pediatric emergency department. Pediatric Emergency Care. 1994; 10(3): 144-9. [PubMed: 8058557]

27. Samuels-Kalow ME, Stack AM, Porter SC. Effective discharge communication in the emergency department. Annals of Emergency Medicine. Aug. 2012; 60(2):152-9. [PubMed: 22221840]

28. Spandorfer JM, Karras DJ, Hughes LA, Caputo C. Comprehension of discharge instructions by patients in an urban emergency department. Annals of Emergency Medicine. 1995; 25(1):71-4. [PubMed: 7802373]

29. Waisman Y, Siegal N, Chemo M, Siegal G, Amir L, Blachar Y, et al. Do parents understand emergency department discharge instructions? A survey analysis. The Israel Medical Association Journa. 2003; 5(8):567.

30. Phillips CO, Wright SM, Kern DE, Singa RM, Shepperd S, Rubin HR. Comprehensive discharge planning with postdischarge support for older patients with congestive heart failure. JAMA: The Journal of the American Medical Association. 2004; 291(11):1358-67.

31. Harrison PL, Hara PA, Pope JE, Young MC, Rula EY. The impact of postdischarge telephonic follow-up on hospital readmissions. Population Health Management. 2011; 14(1):27. [PubMed: 21090991]

32. Joynt KE, Jha AK. A Path Forward on Medicare Readmissions. New England Journal of Medicine. 2013; 368(13):1175-7. PubMed PMID: 23465069. [PubMed: 23465069]

33. Krumholz HM. Post-hospital syndrome-an acquired, transient condition of generalized risk. New England Journal of Medicine. 2013; 368(2):100-2. [PubMed: 23301730] 
- Patients admitted for decompensated heart failure were assessed at discharge

- Patients do not understand many aspects of their discharge, particularly medications

- Patients with perfect discharge comprehension had fewer 30-day readmissions

- The readmission benefit disappeared after controlling for education and home language

- More comprehensive discharge interventions are needed to reduce rates of readmissions 


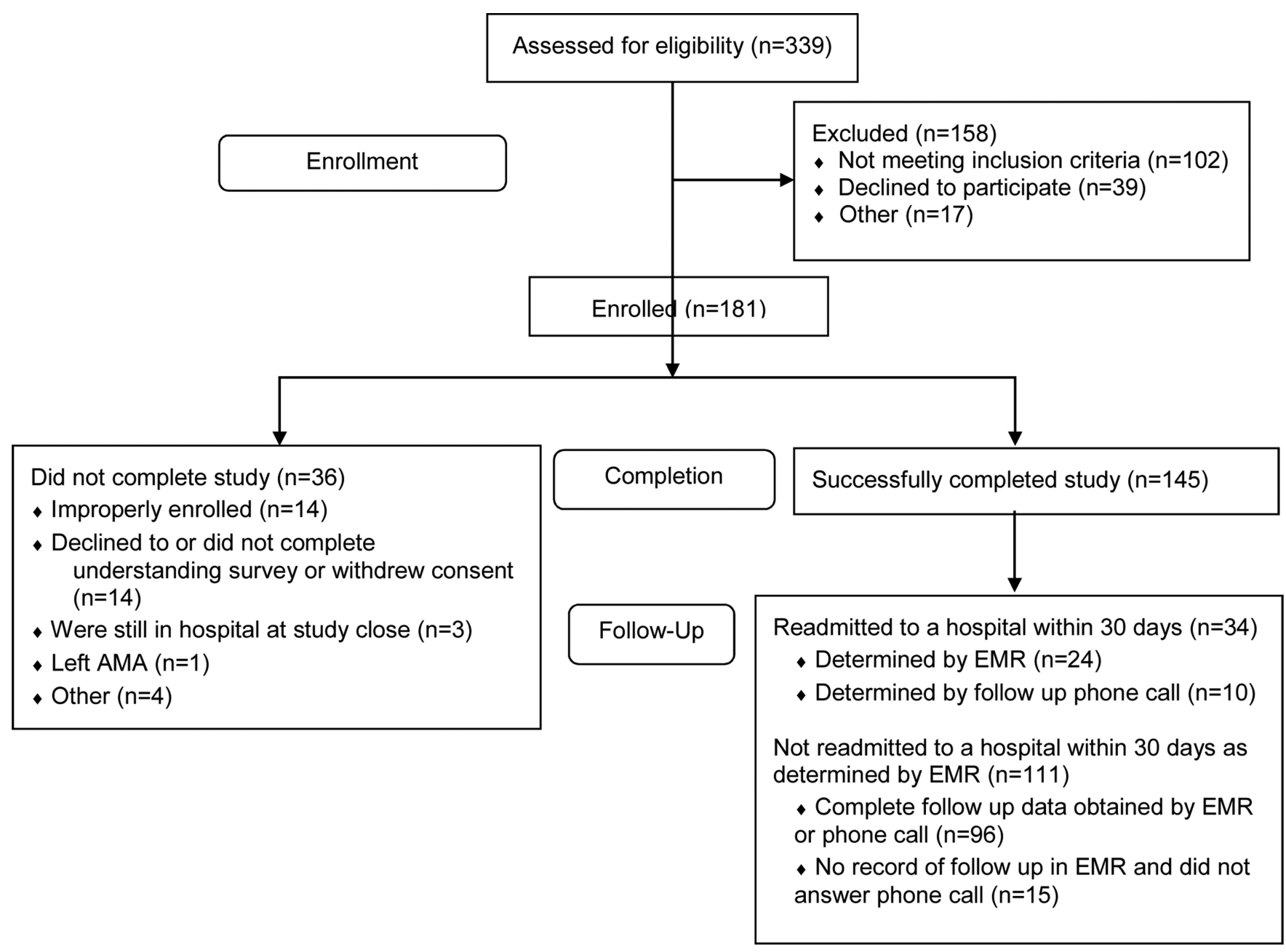

Figure 1.

Study Enrollment and Readmission Results 


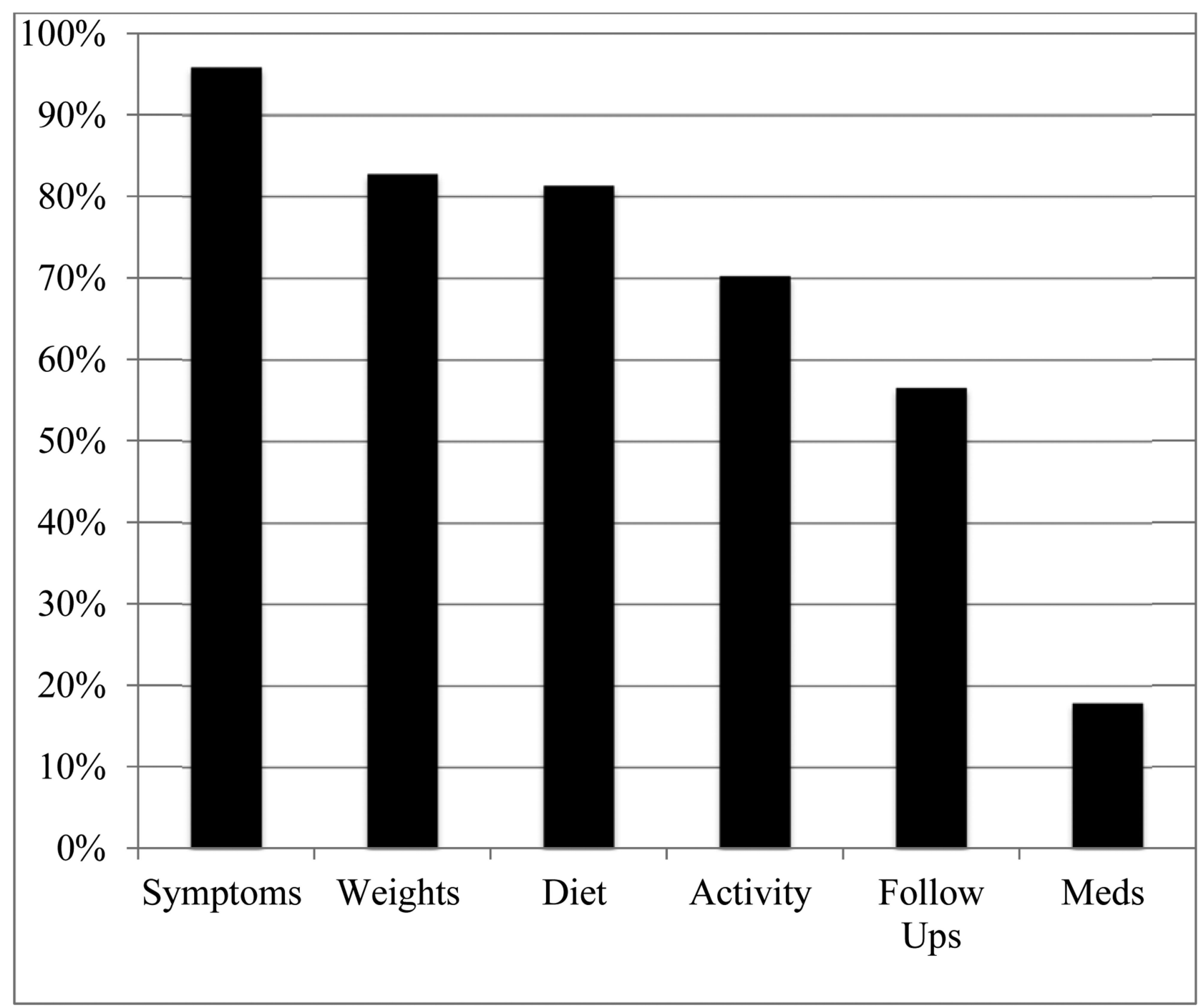

Figure 2.

The percent correct for each of the six individual questions 


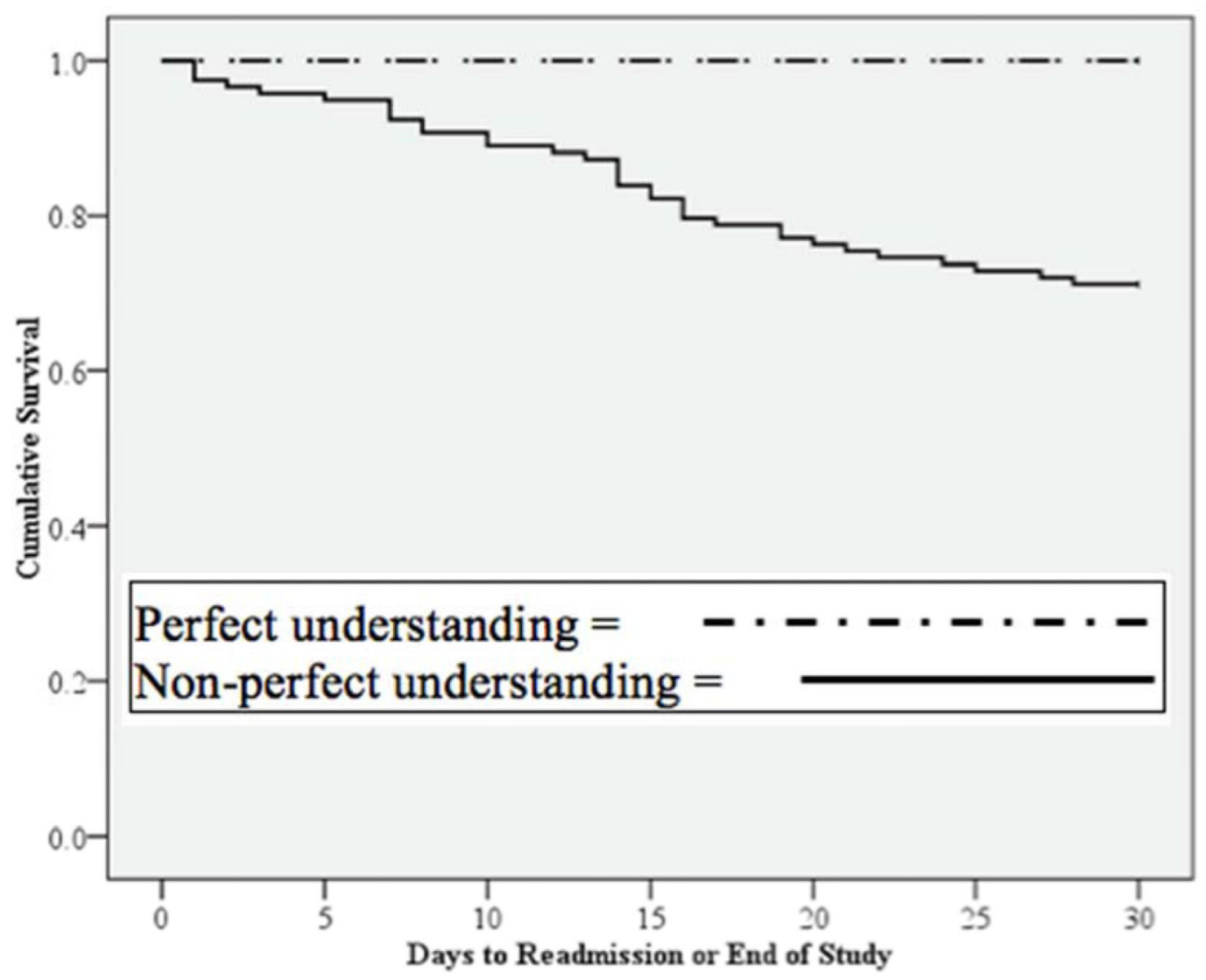

Figure 3.

Kaplan-Meier survival analysis of time-to-hospital-readmission by perfect understanding ( score $=6$ ) versus non-perfect understanding (all other scores), $\mathrm{p}=0.044$ by log rank Mantel-Cox test. 


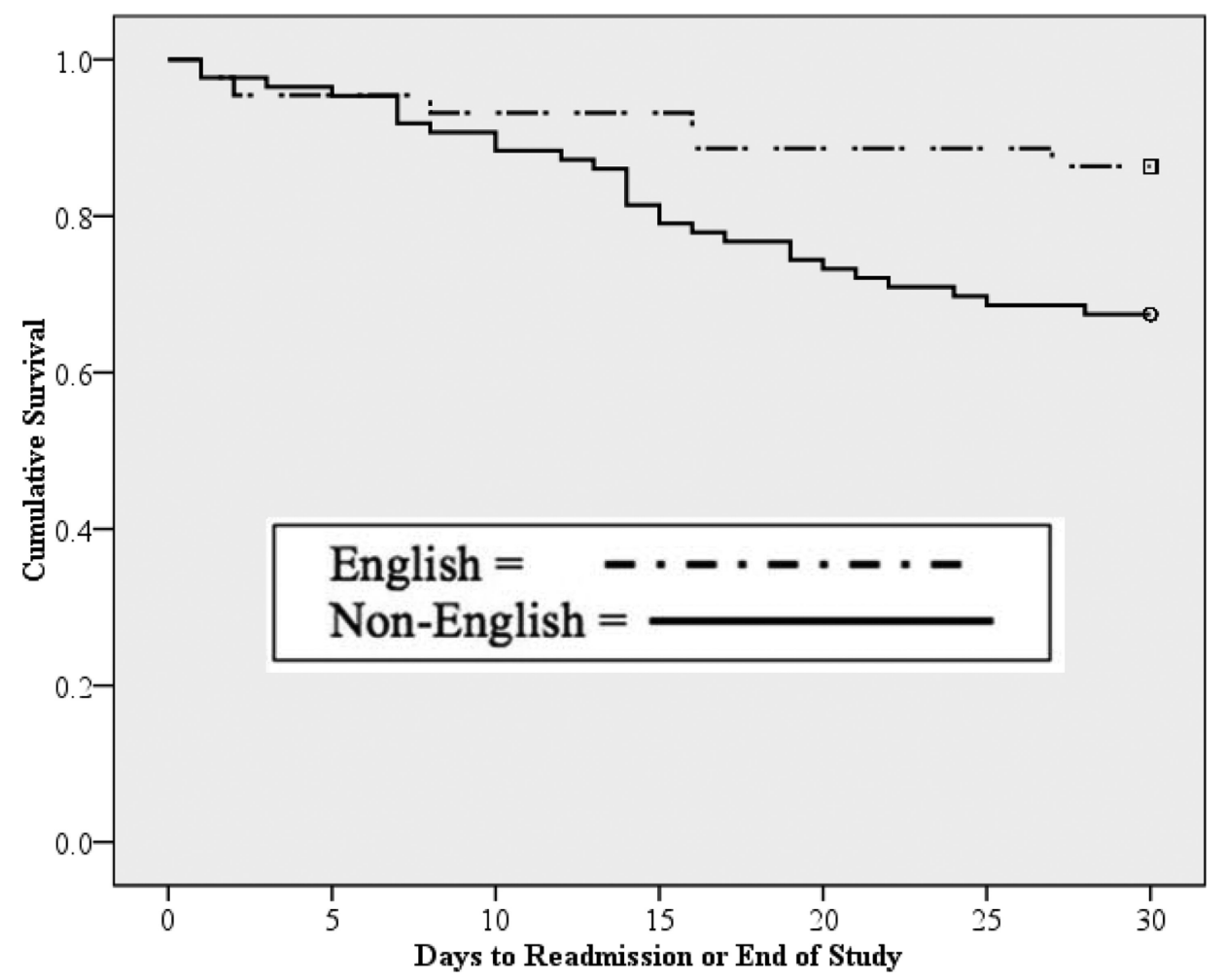

Figure 4.

Kaplan-Meier survival analysis of time-to-hospital-readmission by primary language, $(\mathrm{p}=$ 0.025 ) by log rank Mantel-Cox test. 


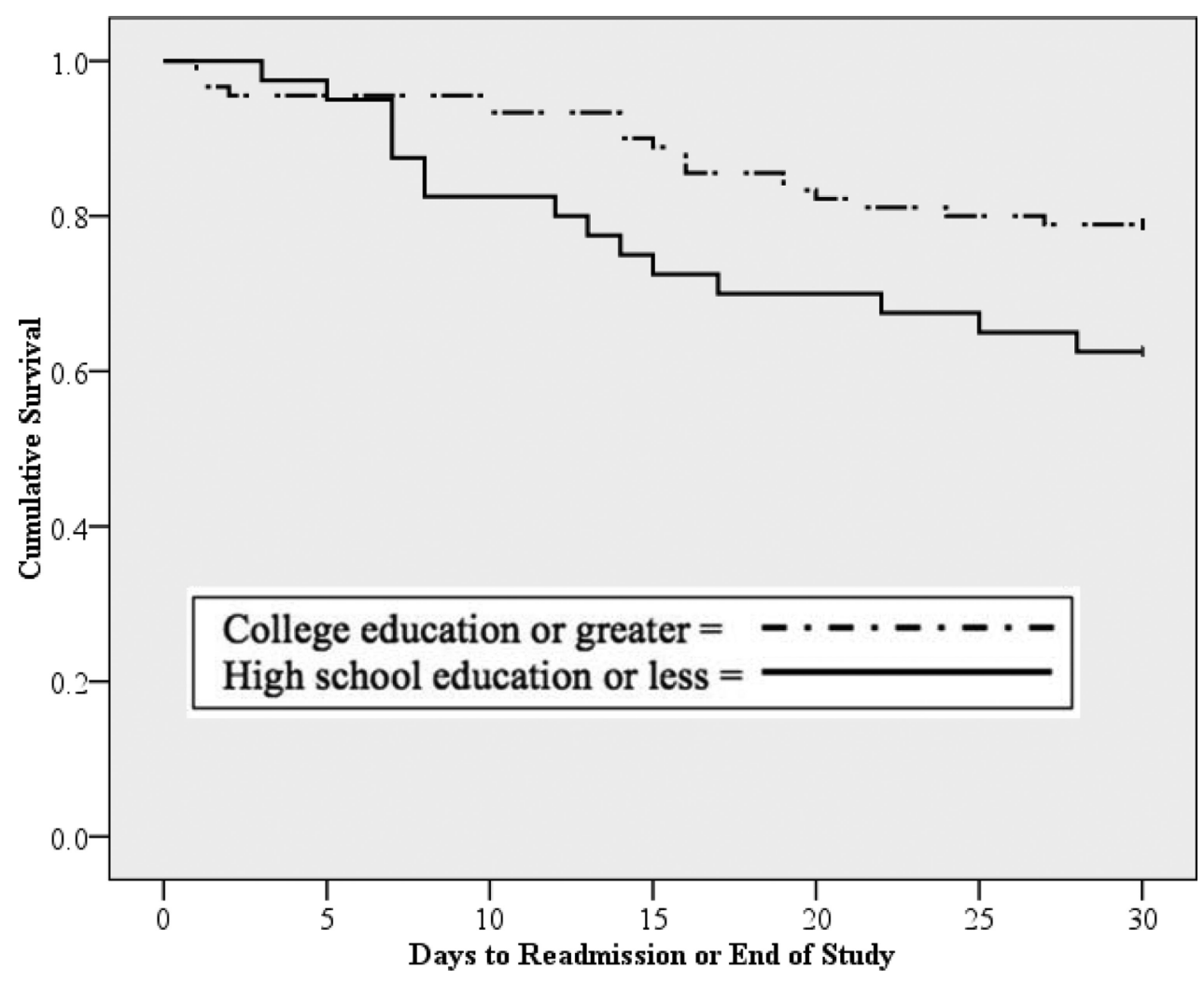

Figure 5.

Kaplan-Meier survival analysis of time-to-hospital-readmission by education level, $(\mathrm{p}=$ 0.039) by log rank Mantel-Cox test. 


\section{Table 1}

Baseline demographic and clinical characteristics of the 145 patients

\begin{tabular}{|c|c|}
\hline \multicolumn{2}{|l|}{ Demographics } \\
\hline Age (mean $\pm \mathrm{SD}$ in years) & $63 \pm 15$ \\
\hline Female & $46 \%$ \\
\hline Married & $42 \%$ \\
\hline White & $32 \%$ \\
\hline Hispanic & $30 \%$ \\
\hline Black & $32 \%$ \\
\hline Other Ethnicity & $7 \%$ \\
\hline \multicolumn{2}{|l|}{ Educational Attainment } \\
\hline High school or less & $67 \%$ \\
\hline College graduate or more & $33 \%$ \\
\hline \multicolumn{2}{|l|}{ Primary Language at Home } \\
\hline English & $69 \%$ \\
\hline Non-English & $31 \%$ \\
\hline \multicolumn{2}{|l|}{ Heart Failure Characteristics } \\
\hline NYHA Class at admission (median) & 3 \\
\hline Ischemic Cardiomyopathy & $41 \%$ \\
\hline LVEF (median) & $28 \%$ \\
\hline $\mathrm{BNP}$ on arrival (median) & 1257 \\
\hline Medications at discharge (mean $\pm \mathrm{SD}$ ) & $9.9 \pm 3.5$ \\
\hline \multicolumn{2}{|l|}{ Comorbidities } \\
\hline Creatinine at discharge (mean $\pm \mathrm{SD}$ ) & $1.4 \pm 0.8$ \\
\hline Charlson Comorbidity Score (mean \pm SD) & $3.6 \pm 1.7$ \\
\hline Hospitalized in past 30 days & $26 \%$ \\
\hline \multicolumn{2}{|l|}{ Hospital Course } \\
\hline Length of Stay (mean $\pm \mathrm{SD}$, days) & $9.4 \pm 6.8$ \\
\hline On a cardiology service & $66 \%$ \\
\hline
\end{tabular}




\section{Table 2}

Average understanding score and breakdown

\begin{tabular}{|c|c|}
\hline Understanding Score & Number of Patients (n=145) \\
\hline 0 & $0(0 \%)$ \\
\hline 1 & $3(2 \%)$ \\
\hline 2 & $11(8 \%)$ \\
\hline 3 & $28(19 \%)$ \\
\hline 4 & $50(35 \%)$ \\
\hline 5 & $39(27 \%)$ \\
\hline 6 & $14(10 \%)$ \\
\hline \multicolumn{2}{|c|}{ Mean $=4.1 \pm 1.2$} \\
\hline
\end{tabular}


Table 3

Effect of clinical variables on discharge understanding score

\begin{tabular}{|l|r|r|}
\hline Clinical Variable & Effect on Understanding Score & Significance \\
\hline English speaker versus non-English speaker & $\mathbf{0 . 8 6}$ & $\mathbf{0 . 0 0 9}$ \\
\hline Hispanic versus non-Hispanic & 0.24 & 0.48 \\
\hline Black versus non-Black & -0.25 & 0.33 \\
\hline College education or more versus high school education or less & $\mathbf{0 . 5 8}$ & $\mathbf{0 . 0 1 2}$ \\
\hline Age (per year increase) & -0.003 & 0.60 \\
\hline Sex (Male versus Female) & 0.11 & 0.58 \\
\hline In CUMC heart failure program & 0.06 & 0.82 \\
\hline Hospitalization in the 30 days prior to index visit & 0.05 & 0.82 \\
\hline On cardiology service & -0.27 & 0.23 \\
\hline Had a heart failure consult & 0.39 & 0.13 \\
\hline PHQ-2 total score & -0.07 & 0.26 \\
\hline
\end{tabular}

Significant variables in bold. Effect on understanding score is unstandardized beta. CUMC $=$ Columbia University Medical Center. PHQ- $2=$ Patient Health Questionnaire-2. 